\title{
Continuous Flow Apparatus for Preparing Isotopically Pure ${ }^{4} \mathrm{He}$
}

\author{
P. C. Hendry and P. V. E. McClintock \\ Department of Physics, \\ University of Lancaster, \\ Lancaster LAl 4YB, UK.
}

\begin{abstract}
A ${ }^{4}$ He isotopic purification cryostat has been developed, capable of sustained operation in continuous flow. Starting from a feedstock of helium of the natural isotopic ratio, ${ }^{3} \mathrm{He} /{ }^{4} \mathrm{He}$ $=x_{3} \approx 10^{-7}$, it yields a purified product for which $x_{3}<5 \times 10^{-13}$ at a production rate of 3.3 STP $\mathrm{m}^{3} \mathrm{~h}^{-1}$. The isotopically purified ${ }^{4} \mathrm{He}$ is being used for a variety of applications, including quantum evaporation experiments, studies of ion motion at the He II/vacuum interface, down-scattering and containment of ultra-cold neutrons, and investigations of the breakdown of superfluidity in ${ }^{4} \mathrm{He}$.
\end{abstract}

Keywords: cryostats; helium; separation techniques; isotopic purification 
Isotopically pure ${ }^{4} \mathrm{He}$ is required for use in a wide range of applications. Of the several cryogenic devices that have been devised for its preparation, all reported to date ${ }^{1-8}$ have employed batch processing techniques of one form or another and they have, therefore, been limited in the quantity of material that could be prepared in the course of one thermal cycle. In some $\operatorname{cases}^{2-5}$ there have also been limitations on the level of isotopic purity that could be attained.

Certain applications require liquid litre quantities of ${ }^{4} \mathrm{He}$ of a quite exceptionally high standard of isotopic purity, the most demanding of all, probably, being the superthermal ultra-cold neutron (UCN) source $^{9}$ which, in the particular case of the Institut Laue-Langevin (ILL) instrument ${ }^{10}$, requires $\sim 15 \mathrm{dm}^{3}$ of liquid ${ }^{4}$ He containing less than a part in $10^{12}$ of ${ }^{3} \mathrm{He}$ (cf. naturally occurring helium with a ${ }^{3} \mathrm{He} /{ }^{4} \mathrm{He}$ ratio of $x_{3} \simeq 10^{-7}$ ). A batch processor is inherently unsuitable for the preparation of ${ }^{4} \mathrm{He}$ of this purity and quantity. Such a purifier would either have to be of enormous physical dimensions or, alternatively, as used until recently at the $\mathrm{ILL}^{8}$, a smaller machine must be thermally cycled several times, making a few liquid Iitres of purified ${ }^{4} \mathrm{He}$ on each occasion. The latter procedure takes a matter of weeks to complete and can be totally vitiated by any hardware failure or operator error that occurs during this period. It must, therefore, be considered rather hazardous and unsatisfactory.

It is against this background that we have been developing what is believed to be the first continuous flow helium isotopic purifier. The instrument to be described below produces ${ }^{4} \mathrm{He}$ in which the ${ }^{3} \mathrm{He}$ content is undetectable, even after reconcentration ${ }^{11}$, and is certainly $<5 \times 10^{-13}$. It is of relatively modest dimensions but, because the product can be extracted continuously, without warming the cryostat, there is effectively no upper limit to the quantity of purified ${ }^{4} \mathrm{He}$ that can be prepared during any one thermal cycle from room temperature. Brief reports on an early prototype ${ }^{12}$, and on the preliminary results obtained from the present purifier $^{13}$, have already been published.

Previous work on helium isotopic separation has been reviewed in an earlier paper ${ }^{1}$ and the discussion need not be repeated here. Since that paper was published, however, two other isotopic purifiers have been reported and should be mentioned. Both are relatively small scale instruments based on the use of superfluid heat flush (see below). First, Yoshiki and his co-workers ${ }^{6}$ have built a prototype purifier, for incorporation into a UCN 
experiment, using a single-shot arrangement with a rouge superleak, rather similar in general design to the purifier previously reported ${ }^{1}$. Second, Moss et al. have developed an interesting variant of the heat flush purifier that is capable of pressurizing a gas bottle at room temperature to $3 \times 10^{6} \mathrm{~Pa}$ with isotopically pure ${ }^{4} \mathrm{He}$, yet manages to avoid inclusion of the usual mechanical valve at low temperature.

\section{Isotopic purification by superfluid heat flush}

The basis of isotopic purification by superfluid heat flush has already been described ${ }^{1}$. It exploits the concentration gradient that develops in a dilute ${ }^{3} \mathrm{He}-{ }^{4} \mathrm{He}$ solution subject to a flux of heat where, as outlined in Figure 1, the corresponding current of normal fluid component tends to flush the ${ }^{3} \mathrm{He}$ atoms away with it. For a semi-infinite flushing tube open at one end to a bath of He II where the ${ }^{3} \mathrm{He}$ concentration is $u(0)$, provided that steady state conditions have been established and provided that no ${ }^{3} \mathrm{He}$ can enter the tube except through its open end, the ${ }^{3} \mathrm{He}$ concentration, $u(x)$, within the tube is given by the one-dimensional form of the equation of gas attack

$$
u(x)=u(0) \exp \left(-v_{n} x / D\right)
$$

where: $x$ is the distance from the mouth of the tube; $v_{n}$ is the normal fluid flow velocity; and $D$ is the coefficient of mass diffusion for the ${ }^{3} \mathrm{He}$. Insertion into Equation (1) of readily attainable values of $v_{n}$ and $x$, together with the values of $D$ measured by Ptukha ${ }^{14}$, demonstrates ${ }^{1}$ immediately that the heat flush phenomenon can provide the basis of an exceedingly powerful technique for isotopic separation.

It is interesting to consider how the efficacy of the technique may be expected to vary with the temperature, $T$, for a given heat flux. Both $v_{n}$ and $D$ will vary rapidly with $T$, but the effects of their temperature dependences on the exponent in Equation (1) will tend to cancel. The normal fluid velocity is given by

$$
v_{n}=\dot{Q} / \rho S T
$$

where: $\dot{Q}$ is the heat flowing per unit area of cross-section; $\rho$ is the bulk liquid density; and $S$ is the entropy per unit mass. If we consider only temperatures $T>1 \mathrm{~K}, S$ can be approximated by the roton entropy ${ }^{15}$ 


$$
S_{r}=\frac{2\left(k_{B} \mu\right)^{1 / 2} p_{0}^{2} \Delta}{(2 \pi)^{3 / 2} \rho \hbar^{3} T^{1 / 2}}\left\{1+\frac{3 k_{B} T}{2 \Delta}\right\} \exp \left(-\Delta / k_{B} T\right)
$$

where $\Delta, P_{0}$ and $\mu$ are the roton parameters. The value of $D$ for the extremely dilute solutions that we are considering is determined for the temperature range in question almost exclusively by interactions between rotons and ${ }^{3} \mathrm{He}$ quasiparticles: it will, therefore, be inversely proportional to the roton number density

$$
N_{r}=\frac{2\left(\mu k_{B} T\right)^{1 / 2} p_{0}^{2}}{(2 \pi)^{3 / 2} \hbar^{3}} \exp \left(-\Delta / k_{B} T\right)
$$

so that

$$
D=\alpha / N_{r}
$$

where $\alpha$ is a constant that can be determined from Ptukha's measurements ${ }^{l 4}$ of $D(T)$. Combining Equations (2)-(5), we can write the coefficient of $x$ in the exponent of Equation (1) as

$$
-\frac{v_{n}}{D}=\frac{-\dot{Q}}{\alpha k_{B}\left(\frac{3}{2} T+\Delta / k_{B}\right)}
$$

which, it may immediately be noted, depends only weakly on $T$ because the exponential terms from Equations (3) and (4) have cancelled. By comparison of Ptukha's measurement of $D$ at $1.4 \mathrm{~K}$ with Equations (4) and (5), incorporating accepted values ${ }^{16}$ of the roton parameters, we find that $\alpha=2.4 \times 10^{19} \mathrm{~m}^{-1} \mathrm{~s}^{-l}$, so that Equation (1) becomes

$$
u=u_{0} \exp -\left\{\dot{Q} x /\left[5.0 \times 10^{-4}(T+5.8)\right]\right\}
$$

where $T$ is in $\mathrm{K}, \dot{Q}$ is in $\mathrm{W} \mathrm{m}^{-2}$ and $x$ is in $\mathrm{m}$. This relation gives the concentration gradient for any given heat flux and temperature. The modulus of the coefficient of $x$ in the exponent, equal $v_{n} n / D$, is plotted as a function of $T$ for various values of $Q$ in Figure 2 .

Erring on the side of prudence, we have adopted as a criterion for satisfactory operation of the purifier that ${ }^{3} \mathrm{He}$ atoms should not be able to back-diffuse more than $1 \mathrm{~mm}$ against the normal fluid wind. The dashed line in Figure 2 corresponds to $u / u_{0}=10^{-30}$ for $x=1 \mathrm{~mm}$ in Equation (7). Provided that the working point lies above this line then, 
in principle, ${ }^{3} \mathrm{He}$ should be eliminated completely from the purified product during its passage through the flushing tube (whose length, in practice, is much greater than $1 \mathrm{~mm}$ : see below).

There are a number of points in relation to the above discussion that deserve further consideration. The first is that the use of Equation (1) for the analysis is valid only under steady state conditions. It is also, of course, essential that $u / u_{0}$ should become effectively equal to zero within a distance which is small compared to the length of the flushing tube, so that the latter can properly be considered semi-infinite. The establishment of steady state conditions after initiation of the normal fluid wind will require at least a few normal fluid transit times. At the higher temperatures, where $v_{n}$ for any given $Q$ becomes relatively small because of the large values taken by $S$ in Equation (2), a significant length of time must elapse before Equation (1) can be assumed to be applicable. Even more important, perhaps, is that, particularly where $v_{n}$ is small, the system will be vulnerable to instabilities of various kinds.

Secondly, it should be noted that our model ${ }^{1}$, in which we considered a gas (the ${ }^{3} \mathrm{He}$ atoms) diffusing within a moving medium (the normal fluid component), is valid only when the ${ }^{3} \mathrm{He} /$ roton mean free path is very small compared to the dimensions of the flushing tube. At low enough temperatures, where the opposite limit will apply, the ${ }^{3} \mathrm{He}$ will behave, instead, like a Knudsen gas in the "mechanical vacuum" of the superfluid component: it will be virtually unaffected by the rarified wind of rotons (and phonons, which, to a first approximation, we can ignore because the ${ }^{3} \mathrm{He}$-phonon scattering crosssection is relatively small) that constitute the current of heat. The problem then no longer involves diffusion of ${ }^{3} \mathrm{He}$ within the normal fluid and the arguments leading to Equation (1) do not apply. We can estimate the temperature at which the transition will occur between these two regimes of behaviour by making use of the approximate expression ${ }^{17}$

$$
D=\left(k_{B} T / M_{3}\right)^{1 / 2}\left(\sigma N_{r}\right)^{-1}
$$

where $M_{3}$ is the ${ }^{3} \mathrm{He}$ atomic mass and $\sigma$ is the roton $-{ }^{3} \mathrm{He}$ scattering cross-section. From a comparison of Equations (5) and (8), and by making use of our numerical estimate of $\alpha$, above, we obtain a value for $\sigma$ of $2.47 \times 10^{-18} \mathrm{~m}^{2}$. The roton- ${ }^{3} \mathrm{He}$ mean free path, $\lambda$, then follows from 


$$
\lambda=\left(\sigma N_{r}\right)^{-1}
$$

Using the above value of $\sigma$ in conjunction with tabulated values ${ }^{16}$ of $N_{r}$ we find that $\lambda=0.4 \mathrm{~mm}$ at $0.5 \mathrm{~K}$, and $=30 \mathrm{~mm}$ at $0.4 \mathrm{~K}$. For a flushing tube with a diameter, typically, of several millimetres, we conclude that Equation (1) will certainly become inapplicable for temperatures significantly below $0.5 \mathrm{~K}$.

Thirdly, the model relates exclusively to the limit where the number density of ${ }^{3} \mathrm{He}$ atoms, $N_{3}$, is very much smaller than $N_{r}$. At low enough temperatures, or large enough values of $N_{3}$, the opposite limit will apply and, again, Equation (1) will be inapplicable. $N_{3}$ for the feedstock of natural helium is $\simeq 4 \times 10^{21} \mathrm{~m}^{-3} ; N_{r}$ is equal to this figure ${ }^{15}$ at $\simeq 0.55 \mathrm{~K}$, and is 10 times greater than it at $0.65 \mathrm{~K}$. So we may regard the criterion of $N_{3} \ll N_{r}$ as being well satisfied for $T>0.7 \mathrm{~K}$. If $N_{3}$ rises above the value for natural helium, then a correspondingly higher temperature will, of course, be required. This latter observation also provides an explanation for the, at first sight, seemingly vexatious question of how a ${ }^{3} \mathrm{He}-{ }^{4} \mathrm{He}$ diiution refrigerator can operate successfully, given that the ${ }^{3} \mathrm{He}$ on the dilute side of the system must diffuse against the normal fluid wind blowing in the tube connecting the still, typically at $0.7 \mathrm{~K}$, to the very much colder mixing chamber. The crucial difference lies in the ${ }^{3} \mathrm{He}$ concentration. For the dilution refrigerator, where the ${ }^{3} \mathrm{He}$ concentration will be a few per cent, the temperature at which $N_{r}=N_{3}$ is shifted well above $1 \mathrm{~K}$. At $0.7 \mathrm{~K}$, therefore, the ${ }^{3} \mathrm{He}$ mean free path is determined mainly by ${ }^{3} \mathrm{He}-{ }^{3} \mathrm{He}$ collisions; ${ }^{3} \mathrm{He}$-roton collisions will be of relatively minor significance; and the model from which Equation (1) is derived will be inapplicable. Thus, although there will be some tendency for the current of normal fluid to reduce the circulation rate of ${ }^{3} \mathrm{He}$ around the system, the circulation will not be prevented from occurring altogether and the refrigerator, therefore, functions as observed in practice.

Fourthly, although we have discussed the purification process in terms of average values of $v_{n}$, it must be borne in mind that $v_{n}$ will not, in fact, be uniform over the cross-section of the flushing tube. The axial velocity profile to be expected is subject to some uncertainty ${ }^{18}$, but it seems likely that $v_{n}$ will be zero at the walls and that it will attain its maximum value on the axis of the tube. Thus, the back-diffusion of ${ }^{3} \mathrm{He}$ will occur, principally, close to the walls. Radial diffusion will also be taking place, however, 
so that, provided that the length of the flushing tube is much greater than both its own radius and also the distance over which $u$ decays effectively to zero (our criterion, above, of $1 \mathrm{~mm}$ ) when calculated on the basis of the average $v_{n}$, the purification scheme should still be efficacious. As an additional safeguard, we have contrived (see below) that there should, in practice, be a radial component of $v_{n}$ towards the axis of the flushing tube over most of its length, so that ${ }^{3} \mathrm{He}$ will continually be carried away from the walls and towards the region of higher axial velocity.

Fifthly, we have so far taken no account of the fact that the He II in the flushing tube will, in reality, be turbulent ${ }^{18}$. Although a tangle of quantized vortex lines represents a collective motion of the superfluid component, the vortex cores effectively scatter the excitations that comprise the normal fluid. As a result, the tangle formed in a simple thermal counterflow tends to drift in the direction of the normal fluid flow ${ }^{19}$, although with a velocity considerably smaller than $v_{n}$. In our case, with an imposed mass flow in addition and hence, effectively, an enhanced value of $v_{s}$, the situation is more complicated. It seems probable, though, that the lines will usually still move in the same direction as $v_{n}$ because the enhancement of $v_{s}$ is normally chosen to be small compared to its absolute value. Whether or not the vortex lines can affect the purification process in reality must depend on the relative magnitudes per unit volume of the net roton $/{ }^{3} \mathrm{He}$ cross-section and the net vortex $/{ }^{3} \mathrm{He}$ cross-section. To estimate the latter quantity we need a value of the equilibrium length, $L_{0}$, of the vortex line per unit volume, given approximately ${ }^{18}$ by

$$
L_{0}^{1 / 2}=1.03 \gamma\left(V-V_{0}\right)
$$

where $\gamma$ is a temperature dependent quantity involving the phenomenological Vinen parameters, for which experimental values have been determined,

$$
V=v_{n}+v_{s}
$$

is the relative velocity of the (usually oppositely directed) normal and superfluid components, and

$$
V_{0} \simeq(\gamma d)^{-1}
$$


where $d$ is the smallest dimension of the flow tube. Taking typical values of $d=16 \mathrm{~mm}$, $Q=2 \times 10^{3} \mathrm{Wm}^{-2}$ and the value ${ }^{18}$ of $\gamma=1.2 \times 10^{6} \mathrm{sm}^{-2}$ at $T=1.4 \mathrm{~K}$ derived from the Schwarz model $^{20}$ of superfluid turbulence, it is straightforward to demonstrate that for this temperature, $L_{0}=10^{10} \mathrm{~m}^{-2}$. If the characteristic length ${ }^{21}$ for the vortex $/{ }^{3} \mathrm{He}$ interaction is $1.8 \mathrm{~nm}$, then the total vortex $/{ }^{3} \mathrm{He}$ cross-section per unit volume for these conditions will be $\simeq 20 \mathrm{~m}^{-1}$. This is to be compared with the total roton $/{ }^{3} \mathrm{He}$ cross-section, $N_{r} \sigma$, which at $1.4 \mathrm{~K}$ is $4 \times 10^{8} \mathrm{~m}^{-1}$. Scattering of ${ }^{3} \mathrm{He}$ by the (slowly moving) vortex lines will thus occur at a rate negligible compared to their scattering by rotons and the ${ }^{3} \mathrm{He}$ will not, therefore, be entrained by the drifting vortex tangle. For temperatures $>0.5 \mathrm{~K}$, the trapping of ${ }^{3} \mathrm{He}$ on vortex lines in our very dilute solutions can also be ignored ${ }^{22}$.

The conclusions to be drawn from these various considerations are that, for effective heat flush isotopic purification of natural helium: 1, the heat flux should be chosen so that the working point lies above the dashed line in Figure 2; 2, the operating temperature must be $>0.7 \mathrm{~K}$; but 3 , to avoid inconveniently long equilibration times and to provide some defence against instabilities, the temperature should not be too close to that of the lambda point, where $v_{n}$ becomes very small. A suitable operating temperature in the range $1.2<T<1.6 \mathrm{~K}$ is readily obtainable with a pumped bath of liquid ${ }^{4} \mathrm{He}$.

\section{Continuous flow ${ }^{4} \mathrm{He}$ isotopic purification}

The majority of the isotopic purification cryostats $^{1-3,5-8}$ previously reported in the literature were based on variants of the design outlined in Figure 3. Helium from a pumped bath of He II (a in Figure 3 ) at $\simeq 1.3 \mathrm{~K}$ is allowed to pass under gravity (except in one case where a fountain pump was used to fill a receiving vessel situated above the main bath) to fill a receiving vessel (b). The earlier purifiers ${ }^{2,3}$ relied entirely upon a superleak (c) to effect isotopic separation and did not necessarily provide an evacuated

space around the product. In later designs ${ }^{1,5-7}$ a source of heat, $\dot{Q}$, was provided just above the superieak to exploit the heat flush purification effect discussed above. In one recent purifier $^{8}$ the superieak was dispensed with altogether; the direction of $V_{n}$ and the bulk flow velocity being controlled, instead, by a needle valve. In no case was provision made for extraction of the product while the purifier was at its operating temperature. It would have been difficult to do so because, with a saturated vapour pressure above the 
product of, at most, a few torr, conventional pressure driven cryogenic transfer techniques could not be used. If two separate (cryogenic) ${ }^{4}$ He baths are provided, however, one of them being at $4.2 \mathrm{~K}$ under atmospheric pressure, with the other at $\simeq 1.3 \mathrm{~K}$ providing the cold base temperature for the heat flush, the problem can readily be overcome because a non-negligible pressure differential can then be established to drive helium continuously round the purification loop.

The basis of operation of the first such continuous flow purifier to be reported ${ }^{12}$ is sketched in Figure 4. Liquid helium from a bath (a in Figure 4) under atmospheric pressure at $4.2 \mathrm{~K}$ passes down a cupro-nickel concentric tube heat exchanger (b) and enters the flushing tube (c) close to its colder end, adjacent to a pumped pot (d) of liquid ${ }^{4} \mathrm{He}$ at $\simeq 1.3$ $\mathrm{K}$ with which the flushing tube is in thermal contact. The main flow in the flushing tube is oppositely directed to the normal fluid flow induced by the heat input $(\dot{Q})$ : a superleak (e) in the flushing tube serves to block any flow of normal fluid in the direction of the mass flow. The purified product passes up the other side of the heat exchanger (b) and exits from the cryostat through the outlet tube (f). The liquid within the purification loop is all approximately at atmospheric pressure, but the small pressure differential induced by pumping the outlet is sufficient to drive helium continuously through the system. This device was moderately successful in that, after various modifications to the flushing tube, it produced ${ }^{4} \mathrm{He}$ in which the ${ }^{3} \mathrm{He}$ was undetectable $\left(<2 \times 10^{-10}\right)$. On other occasions, however, a product purity of only $2 \times 10^{-9}$ was attained. The reasons for the inconsistent behaviour are unknown for certain, but they are believed to have been associated with instabilities (at the outlet) that prevented steady state conditions from being maintained within the flushing tube.

The purifier design illustrated in Figure 4 is inherently inefficient because, as a result of the presence of the superleak, the heat flowing down the moving columns of liquid in the heat exchanger is all transferred to the colder end of the flushing tube. It does not, therefore, contribute significantly to the purification process. Furthermore, the huge surface area and long out-gassing time of the superleak itself provide a potential source of contamination if it should at any time inadvertently be exposed to natural helium.

These problems have all been overcome in the modified design that we have now evolved, which is outlined very diagrammatically in Figure 5. The incoming stream of 
helium from the main bath (a in Figure 5) still enters the flushing tube (c) at its colder end, but the associated flux of heat enters the flushing tube at its warmer end. This is accomplished by making the flushing tube of copper and providing it with a closely fitting copper jacket through which the incoming stream of helium passes. There is no superleak. With this arrangement, all of the heat leaking down the cupro-nickel heat exchanger, regardless of whether it enters through the inlet or outlet side, eventually passes through the inside of the flushing tube along most of its length, increasing the average axial normal fluid velocity and thereby enhancing the efficiency of the purification process. The second advantage to be derived from this particular arrangement is that some normal fluid will effectively be entering the flushing tube radially through its walls. This helps to prevent ${ }^{3} \mathrm{He}$ atoms becoming trapped or back-diffusing (see above) within the boundary layer, where the axial component of $v_{n}$ tends to zero.

\section{Continuous flow isotopic purifier}

In reality, the continuous flow purifier is rather more complicated than the sketch of Figure 5 would appear to imply. This is because, in particular, it has been necessary to make provision for the removal of extracted ${ }^{3} \mathrm{He}$ from the flushing tube; it has also been important to minimize the thermal resistance between the liquid helium in the purification circuit and that in the pumped pot. Both of these requirements are met by the inclusion of a conical copper heat exchanger of the type discussed previously ${ }^{1}$.

A more detailed schematic diagram of the purifier, and a photograph of the insert with the vacuum can removed, are shown in Figures 6 and 7, respectively. Circulation of helium through the purification loop occurs in the manner already described, with isotopic separation occurring within the flushing tube (c in Figure 6). The latter consists of a 2 m length of $14 \mathrm{~mm}$ i.d., $16 \mathrm{~mm}$ o.d. copper tube. To enable it to fit within the vacuum can, it is divided into six separate lengths, connected by wide-bore junction pieces. The encircling sleeves on the individual sections of flushing tube are of $17 \mathrm{~mm}$ i.d., $19 \mathrm{~mm}$ o.d. copper, connected together by short lengths of $3 \mathrm{~mm}$ o.d. cupro-nickel tube. The concentric tube heat exchanger ( $\mathrm{f}$ ) is made up from a $3 \mathrm{~m}$ length of $3 \mathrm{~mm}$ o.d. cupro-nickel tubing inside $5 \mathrm{~mm}$ o.d., $0.2 \mathrm{~mm}$ wall, cupro-nickel tubing.

The pumped helium bath consists of a $38 \mathrm{~mm}$ i.d. stainless steel tube, closed at 
the bottom by the wide end of the copper cone (l) alluded to above. This bath can be filled continuously: either through the part-closed needle valve $\left(\mathrm{g}_{3}\right)$ from the main helium reservoir; or through needle valve $\mathrm{g}_{4}$ via a restriction $(\mathrm{k})$ with slightly ${ }^{3} \mathrm{He}$-enriched liquid helium from within the narrow end of the cone. The restriction consists of $\simeq 350 \mathrm{~mm}$ of nominally $0.3 \mathrm{~mm}$ i.d. cupro-nickel, this length being chosen such that its impedance is suitable for maintenance of the helium level within the bath of He II (d) under steady state circulation. The liquid level within the bath is monitored by a capacitance depth gauge. The bath is filled initially through $\mathrm{g}_{3}$ which is subsequently closed off again once normal operation has been established. It is pumped through a $100 \mathrm{~mm}$ i.d. pumping line by an Edwards EH1200 mechanical booster, backed by a 1SC900 rotary pump.

To monitor the operation of the purifier, carbon resistance thermometers are positioned just above and just below the cone, and at various positions along the flushing tube. Provision is made for the application of heat to the warm end of the flushing tube and directly to the pumped bath, using the heaters $\mathrm{q}_{1}$ and $\mathrm{q}_{2}$ (in Figure 6).

The isotopically purified liquid ${ }^{4}$ He leaves the cryostat through the exit tube (f) where it enters a standard helium transfer siphon. The liquid is evaporated in a heated copper ballast volume at room temperature, passes through the small rotary pump that is used to induce the flow, and enters a $4.5 \mathrm{~m}^{3}$ flexible reservoir. At intervals, helium from the latter is compressed into a bank of standard gas bottles, to a maximum pressure of 1800 psi, by means of a Williams and James Triquad compressor. The latter has been modified slightly so that gas from the stage unloaders is returned to the laboratory helium recovery system but, otherwise, is exactly as provided by the manufacturers.

In an earlier version of the purifier, provision was made for collection of the extracted ${ }^{3} \mathrm{He}$ in a small copper pot immersed within the helium in the pumped bath ( $\mathrm{d}$ in Figure 6 ) in place of the present restriction $(\mathrm{k})$. The ${ }^{3} \mathrm{He}$-enriched product was extracted via the guide tube $(\mathrm{p})$ leading to needle valve $\mathrm{g}_{4}$. This arrangement had the disadvantage that, when the system was closed down overnight with $\mathrm{g}_{4}$ closed, while being kept at $4 \mathrm{~K}$ ready for a resumption of production on the following day, any small leak in $\mathrm{g}_{4}$ would have enabled the collected ${ }^{3} \mathrm{He}$ to leak back into the flushing tube. While any such inadvertent contamination of the system should have cleared rapidly when the heat flush was reestablished, it was felt to be undesirable. The quantities of ${ }^{3} \mathrm{He}$ collected were, in any 
case, small. In the present version of the purifier, extracted ${ }^{3} \mathrm{He}$ is removed continuously through the capillary restriction $(\mathrm{k})$; no significant ${ }^{3} \mathrm{He}$ enrichment occurs, therefore, in any part of the system during operation.

\section{Operation of the purifier}

The operating procedure used for the purifier, in outline, is as follows. The device is leak tested at room temperature and at $77 \mathrm{~K}$; its dewar is filled with liquid helium; thermal exchange gas in the vacuum space ( $\mathrm{j}$ in Figure 6 ) is pumped out; the bath $(\mathrm{d})$ is filled through needle valve $g_{3}$; it is then pumped towards $1.2 \mathrm{~K}$ while, at the same time, the carbon resistance thermometers are calibrated against ${ }^{4} \mathrm{He}$ vapour pressure. The needle valve $g_{1}$ is then opened, admitting liquid helium to the flushing tube and cone.

At this stage, even in the absence of fluid flow through the purification system, there is a large static heat load on the pot (d) most of which arises from conduction through the columns of liquid in the concentric tube heat exchanger (b). The heat load becomes even larger once flow has been initiated, but admission of liquid via the restriction and valve $\mathrm{g}_{4}$ is sufficient to maintain an approximately constant level within the He II bath (d). On first starting the flow, the pressure at the room temperature end of the siphon in the exit tube (f) undergoes large fluctuations, probably corresponding to the initial cooling down of the inside of the siphon. After a few minutes, however, the system settles down to a steady state with a flow of $3.3 \mathrm{~m}^{3} \mathrm{~h}^{-1}$ (the speed of the small rotary pump that draws gas through the system) of gas at STP. The pressure difference between the main ${ }^{4} \mathrm{He}$ both at $4.2 \mathrm{~K}$ and the outlet of the exit siphon settles at $\simeq 10^{4} \mathrm{~Pa}$. The first few cubic metres of isotopically purified product are used to purge air and other contaminants from the rotary pump, compressor, reservoir, bottles and gas handling system; collection and storage of the isotopically purified ${ }^{4} \mathrm{He}$ commences once the purifier has been running in a steady state for $\simeq 1 \mathrm{~h}$.

It is of interest to try to establish the magnitude of the heat current in the flushing tube, to ensure that the operating point of the system lies well above the dashed line of Figure 2. An a priori calculation, based on the dimensions of the system, would be extremely difficult on account of the complicated nature of heat flow in He II; and particularly given that there are continuous columns of liquid extending from a region at 
$1.2 \mathrm{~K}$ to one at $4.2 \mathrm{~K}$, with $T_{\lambda}$ somewhere in the concentric tube heat exchanger. We can, however, make an experimental estimate of the heat current, by measurement of the Kapitza temperature discontinuities at the copper cone. The temperature difference, $\Delta T$, across the cone is in practice, small, so we may assume the two discontinuities are approximately equal. Then

$$
\Delta T=2\left(K_{R} / A\right)\left(\dot{Q}_{0}+\dot{Q}_{1}\right)
$$

where: $K_{R}$ is the Kapitza resistance; $A$ is the surface area of one side of the cone; $\dot{Q}_{0}$ is the heat current arising through conduction from the main helium reservoir; and $\dot{Q}_{1}$ is the imposed heat current applied by energizing the heater $\left(\mathrm{q}_{1}\right)$. The precise value of $K_{R}$ for the (probably highly oxidized) surfaces of the copper cone is subject to considerable uncertainty, but we can establish $Q_{0}$ by measurement of $\Delta T$ for a range of values of $\dot{Q}_{1}$, as shown in Figure 8. These measurements were made in the absence of circulation, with the He II bath (d) maintained at a constant temperature by adjustments to the power dissipated in a second heater $\left(\mathrm{q}_{2}\right)$ positioned above the cone. From Equation (13) it is clear that the graph should be a straight line whose abscissa intercept yields directly a value of $-Q_{0}$. From a number of plots, such as that in Figure 8, fitting straight lines to the data by the method of least squares, we have concluded that the static heat load on the pot is

$$
\dot{Q}_{0}=(0.8 \pm 0.1) \mathrm{W}
$$

The quoted uncertainty probably reflects a variation in the absolute value of $\dot{Q}_{0}$, corresponding to the gradual decrease in the temperature of the main helium reservoir that took place between helium fills. The derived value of $\dot{Q}_{0}$ is consistent with the measured evaporation rate from the He II bath (d) with needle valves $g_{3}$ and $g_{l}$ closed off.

The heat current, $\dot{Q}_{F}$, in the presence of a mass flow was estimated in a similar manner. Under standard operating conditions, with a throughput of $3.3 \mathrm{STP} \mathrm{m}^{3} \mathrm{~h}^{-1}$, the heat current in the flushing tube was found to be

$$
\dot{Q}_{F}=(1.6 \pm 1.1) \mathrm{W}
$$


The corresponding temperature gradient along the flushing tube was extremely small, the temperature difference between its ends being $10 \pm 2 \mathrm{mK}$.

It is evident that, even with the static heat load alone, the purifier functions within the region of safe operation, well above the dashed line in Figure 2; under steady flow conditions, the heat flux close to the bottom of the cone is $\simeq 10^{4} \mathrm{Wm}^{-1}$, equivalent to a point close to the top of the figure. It seems unlikely, therefore, that any ${ }^{3} \mathrm{He}$ is able to enter the flushing tube itself. Rather, it will be flushed straight up into the cone as soon as it emerges from the inlet tube from the sleeve and enters the normal fluid current. The provision of a long flushing tube is, however, a valuable guard against inadvertent contamination of the product as a consequence of the mechanical instabilities that occur during the start-up period and that may also occur when any of the operating parameters is changed.

\section{Results}

Samples taken from the gas bottles after completion of a production run were analysed by mass spectrometry. The ${ }^{3} \mathrm{He}$ content was found to be undetectable, implying that $x_{3}<2 \times 10^{-10}$. Further samples of the gas were then reconcentrated in the miniature isotopic concentrator already described ${ }^{11}$. This procedure would have enhanced the concentration of any ${ }^{3} \mathrm{He}$ that was present by a factor of almost 500. A sample of the resultant concentrate was sent for analysis and, again, it was found that the ${ }^{3} \mathrm{He}$ content was undetectable. We conclude, therefore, that the isotopic ratio of the $4 \mathrm{He}$ from the purifier was $<5 \times 10^{-13}$.

These results are consistent with our belief, based on the use of Equation (1), that the helium emerging from the purifier is devoid of ${ }^{3} \mathrm{He}$; but there is, of course, no means of proving that this is in fact the case.

Helium from the purifier (or from its earlier variants) has already been employed successfully for experiments on quantum evaporation ${ }^{23}$, on the cyclotron resonance of ions at a He II surface ${ }^{24}$, and on the downscattering and containment of ultra-cold neutrons ${ }^{25}$, as well as in support of the Lancaster research programme on ${ }^{4} \mathrm{He}$ superfluidity ${ }^{26-28}$ for which the device was originally developed. 


\section{Conclusions}

The ${ }^{4} \mathrm{He}$ isotopic purification cryostat that we have described is capable of producing, in continuous operation and therefore in virtually unlimited quantities, a product whose isotopic ratio is certainly $<5 \times 10^{-13}$ and, in reality, probably a great deal smaller than this. It is, therefore, capable of supporting even the most demanding of the applications of isotopically purified ${ }^{4} \mathrm{He}$ that has yet been devised.

\section{Acknowledgements}

The work was supported in part by the University of Lancaster Research Fund and by the Science and Engineering Research Council. We are grateful to Mr D. E. Emerson of the US Bureau of Mines, Amarillo, Texas, USA, for the mass spectrometric analyses of several helium samples.

\section{References}

1. McClintock, P.V.E. Cryogenics (1978) 18201.

2. Mezhov-Deglin, L.P. Prib Tekh Eksper (1971) 3 217; Cryogenics (1972) 12311.

3. Fatouros, P.P., Edwards, D.O., Gasparini, F.M. and Shen, S. Y. Cryogenics (1975) 15147.

4. Tully, P.C. US Bureau of Mines Report of Investigations 8054, Washington DC, USA (1975).

5. Atkins, M. and McClintock, P.V.E. Cryogenics (1976) 16733.

6. Yoshiki, H., Morimoto, K., Kudo, N. and Kiyanagi, Y. Proc 35th Annual Meeting Japanese Phys Sac (1980) paper 27aPI; Yoshiki, H. KENS Report V (Ed Ishikawa, Y. et al.) KEK (1984) 0215; Yoshiki, H. KEK Report 84-2 (1984) in Japanese; Yoshiki H. personal communication (1985). (The KEK reports are available from Technical Information Office, National Laboratory for High Energy Physics, OhoMaehi, Tsukubagun, Ibaraki-ken, 305, Japan)

7. Moss, F.E., Wyatt, A.F.G. and Baird, M.J. Cryogenics (1981) 21114.

8. Jewell, C.I., Golub, R. and McClintock, P.V.E. Cryogenics (1982) 22373.

9. Golub, R. and Pendlebury, J.M. Phys Lett A (1977) 62337.

10. Golub, R., Jewell, C.I., Ageron, P., Mampe, W., Heckel, B. and Kilvington, L. Z 
Phys B (Condensed Matter) (1983) 51187.

11. Hendry, P.C. and McClintock, P.V.E. in: Proc 17th Int Conf Low Temp Phys (Eds Eckern, U., Schmid, A., Weber. W. and Wuhl, H.) North Holland, Amsterdam, The Netherlands (1984) Part 1, 417.

12. Ngan, T.H., Small, J.C.H. and McClintock, P.V.E. Proc 16th Int Conf Low Temp Phys (Ed Clark, W.G.) North Holland, Amsterdam, The Netherlands (1981) 597.

13. Hendry, P.C. and McClintock, P.V.E. Cryogenics (1985) 25526

14. Ptukha, T.P. Zh Eksp Tear Fiz (1961) 401583 (Soviet Phys JETP (1961) 131112 ).

15. Wilks, J. The Properties of Liquid and Solid Helium, Clarendon Press, Oxford, UK (1967).

16. Brooks, J.S. and Donnelly, R.J., J Phys Chem Ref Data (1977) 651.

17. Khalatnikov, I.M. An Introduction to the Theory of Superfluidity Benjamin, New York, USA (1965).

18. Tough, J.T. in: Progress in Low Temperature Physics Vol VIII (Ed. Brewer, D.F.) North Holland, Amsterdam, The Netherlands (1982) 133.

19. Ashton, R.A. and Northby, J.A. Phys Rev Lett (1975) 351714.

20. Schwarz, K.W. Phys Rev B (1978) 18245.

21. Rayfield, G.W. and Reif, F. Phys Rev (1964) 136 A1194.

22. Ostermeier, R.M. and Glaberson, W.I. J Low Temp Phys (1976) 25317.

23. Baird, M.J., Hope, F.R. and Wyatt, A.F.G. Nature (1983) 304325.

24. C.F. Barenghi, C.J. Mellor, C.M. Muirhead and W.F. Vinen J Phys C (1986) 19 1135.

25. Jewell, C.I. personal communication (1986).

26. Bowley, R.M., McClintock, P.V.E., Moss, F.E., Nancolas, G.G. and Stamp, P.C.E. Phil Trans R Soc (London) A (1982) 307201.

27. Nancolas, G.G., Bowley, R.M. and McClintock, P.V.E. Phil Trans R Soc (London) A (1985) 313537.

28. Ellis, T. and McClintock, P.V.E. Phil Trans R Soc (London) A (1985) 315259. 


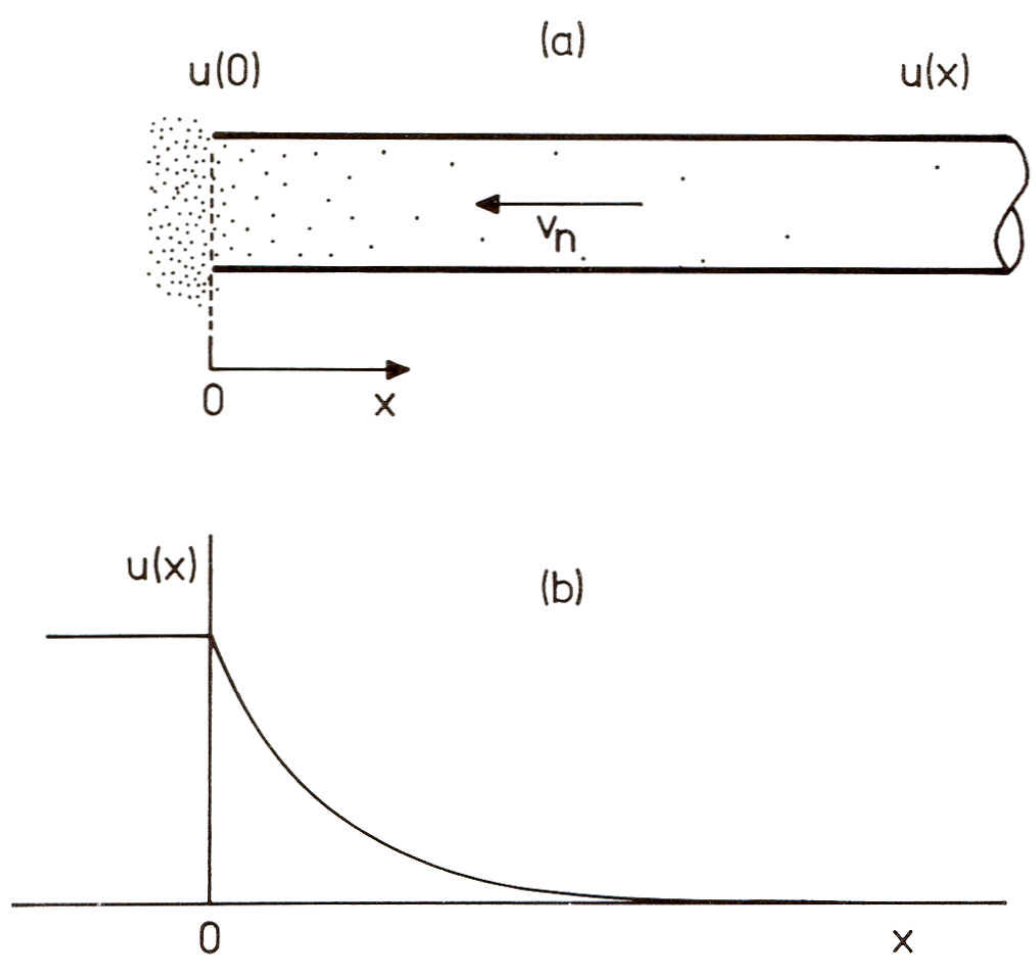

Figure 1: Diagram to illustrate the principle of heat flush ${ }^{4} \mathrm{He}$ isotopic purification. (a) A semi-infinite tube is open at one end to a bath of He II containing a small concentration $u(O)$ of ${ }^{3} \mathrm{He}$ impurity atoms, indicated as dots. If there is a normal fluid flow at velocity $v_{n}$ out of the tube, this will limit the average distance through which the ${ }^{3} \mathrm{He}$ atoms are able to back-diffuse into the tube, as described quantitatively by Equation (1). (b) Sketch of the variation of ${ }^{3} \mathrm{He}$ concentration, $u(x)$, with distance $x$ from the end of the tube. In practice, the operating parameters are chosen such that $u(x)$ falls effectively to zero at $x \leq 1 \mathrm{~mm}$. 


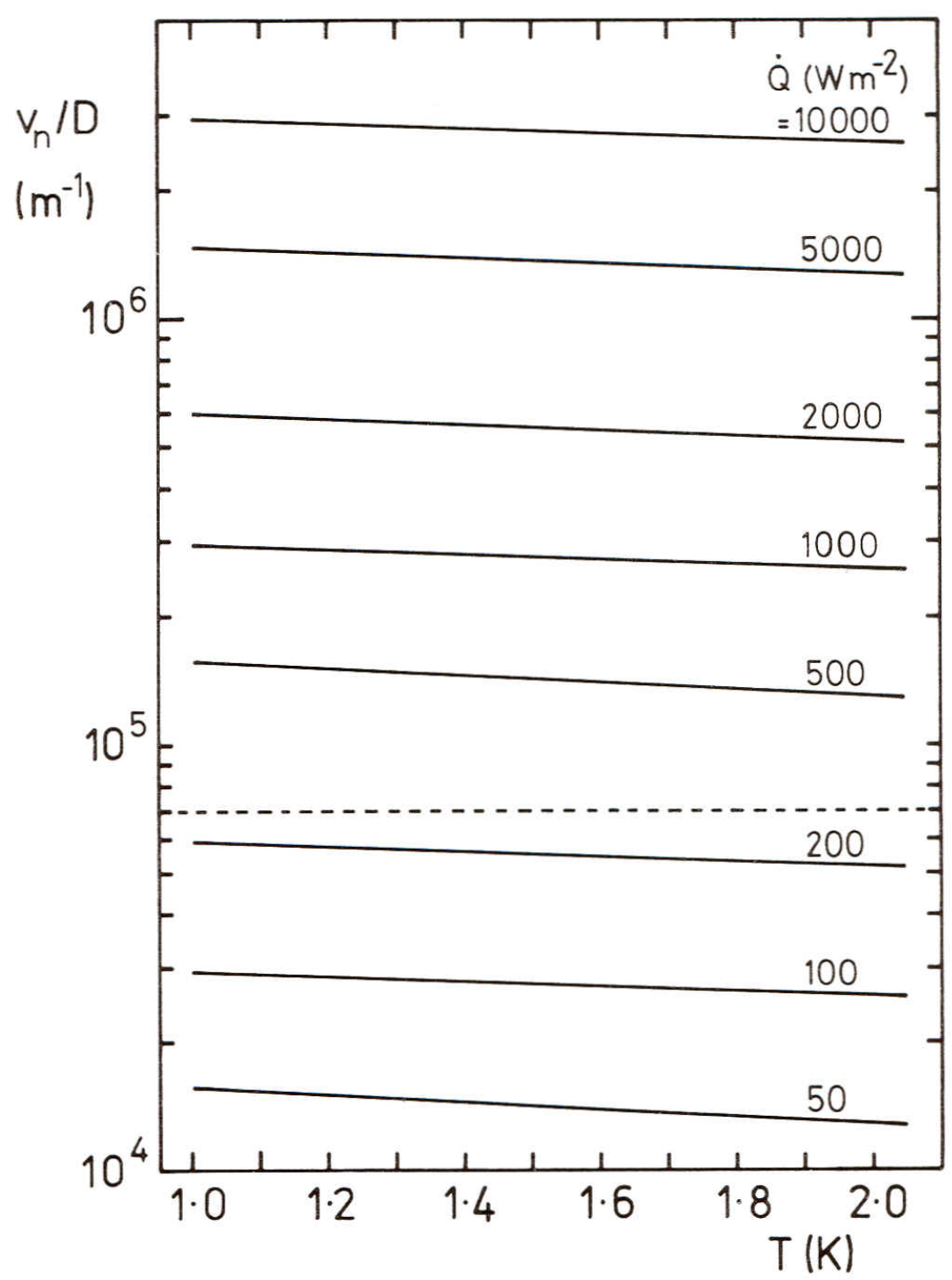

Figure 2: Modulus of the coefficient of $x$ in the exponent of Equation (1), calculated from the approximate expression (6), by use of accepted values of the roton parameters and the coefficient of mass diffusion of ${ }^{3} \mathrm{He}$ in He II. The different curves refer to a range of values of the heat current density, $\dot{Q}$. as indicated. An isotopic purifier should functio.n reliably if its operating point lies above the dashed line (see text). 


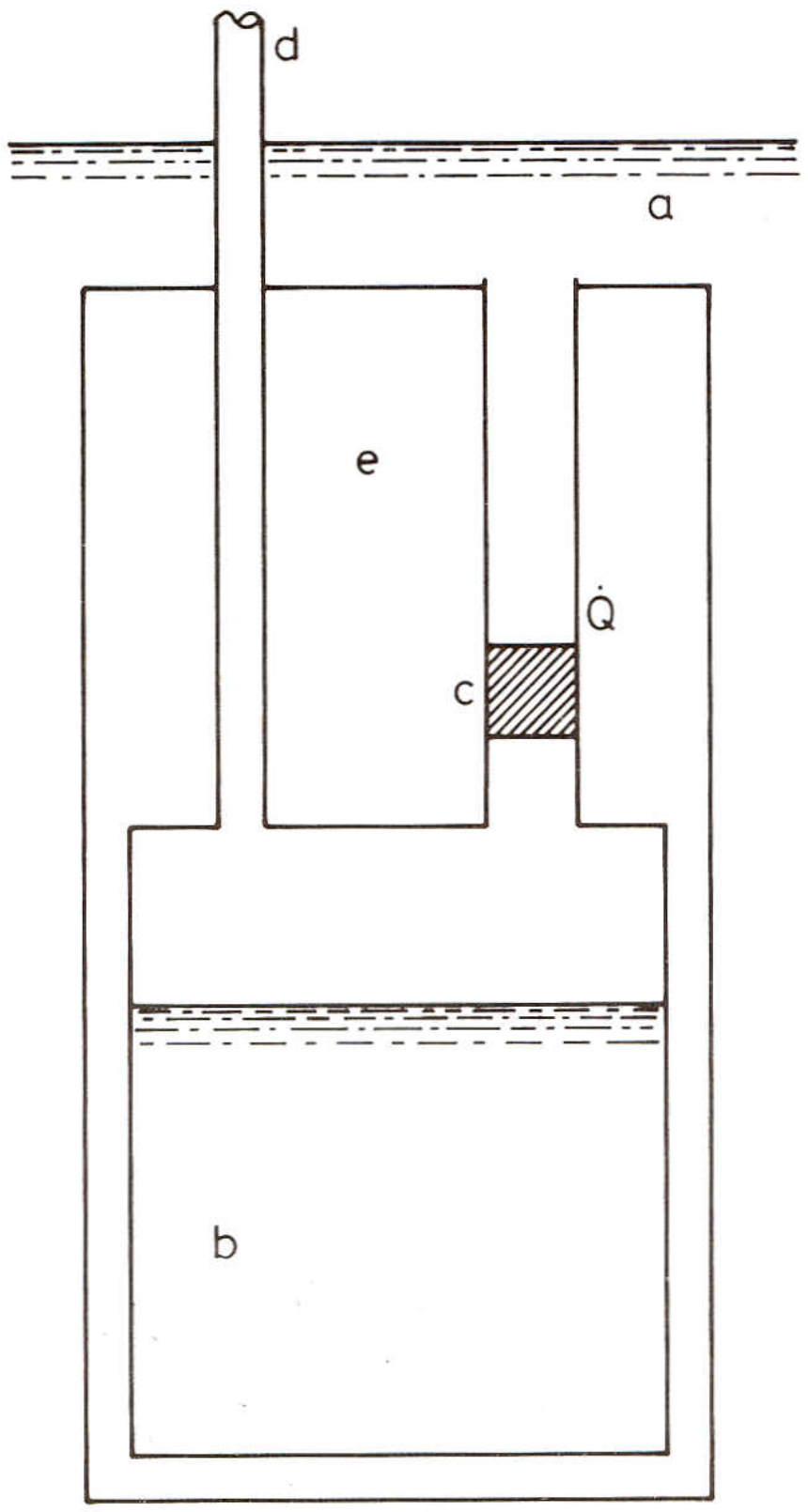

Figure 3: ${ }^{4} \mathrm{He}$ isotopic purification by simple flow through a superleak. a, Bath of He II of natural isotopic composition; b, isotopically purified ${ }^{4} \mathrm{He}$; c, superleak (e.g. compressed rouge or Vycor glass); d, exit tube for the purified product; e, vacuum. 


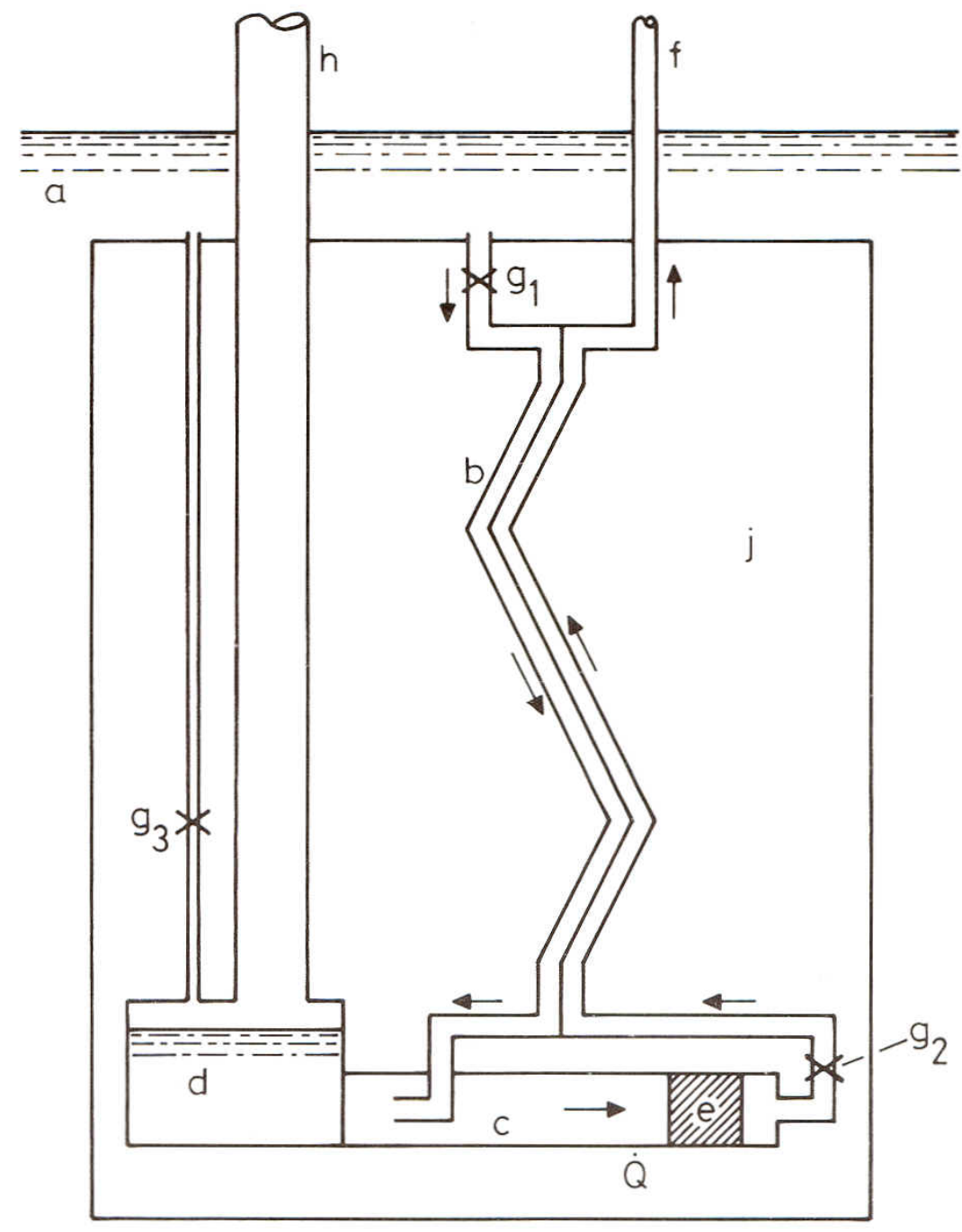

Figure 4: Diagram to illustrate the working principles of the prototype continuous flow heat flush isotopic purification cryostat. a, Bath of liquid helium of natural isotopic ratio at $4.2 \mathrm{~K}$; b, concentric tube heat exchanger; c, flushing tube; d, pumped bath of He II at $1.2 \mathrm{~K}$; e, rouge superleak; f, exit tube for isotopically purified ${ }^{4} \mathrm{He} ; \mathrm{g}_{1} \mathrm{~g}_{2}, \mathrm{~g}_{3}$, needle valves; h, pumping tube for bath, $\mathrm{d} ; \mathrm{j}$, vacuum space; $\dot{Q}$, heat input to create normal fluid flow. The directions of mass flow are indicated by the arrows. 


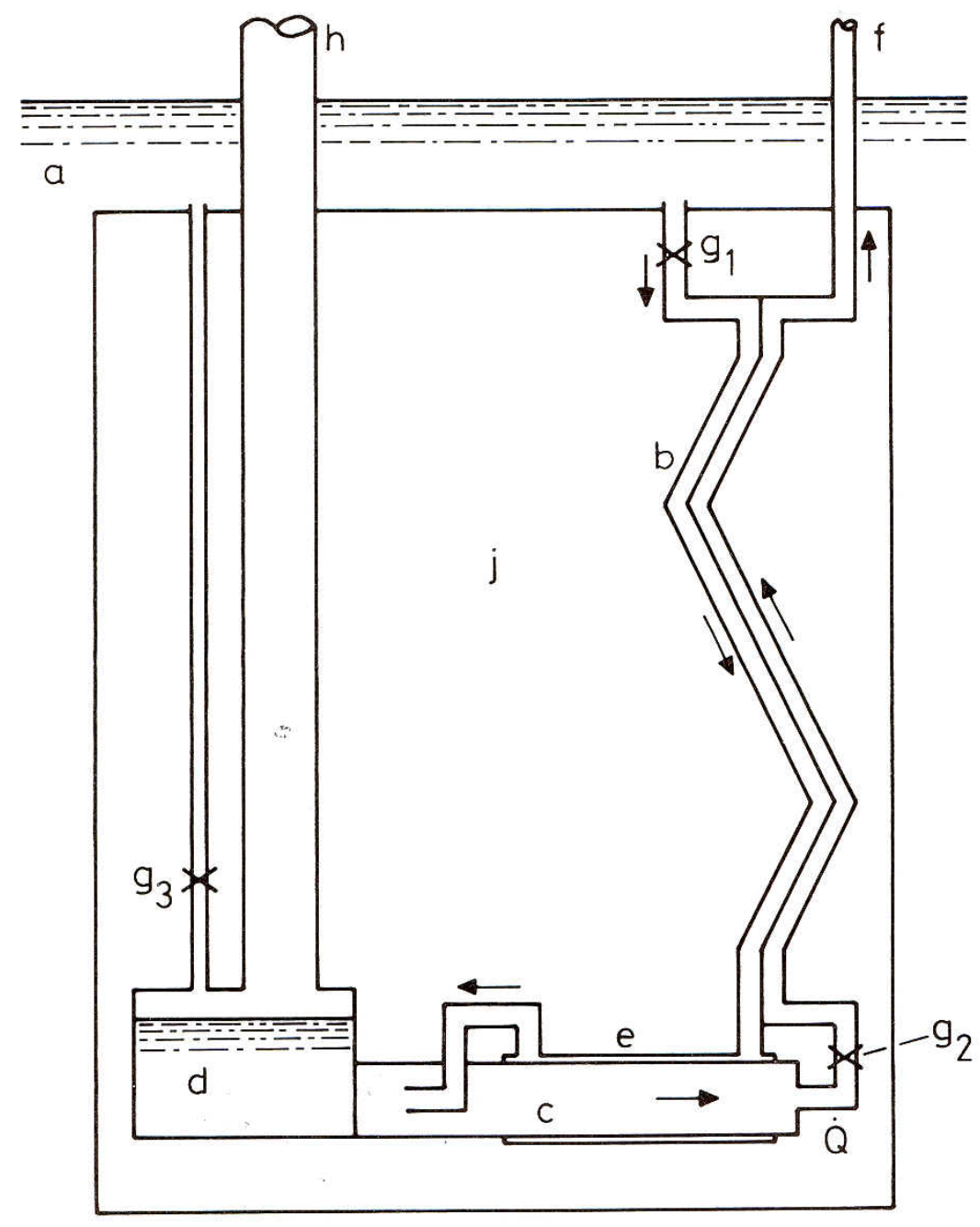

Figure 5: Diagram to illustrate the working principles of the present isotopic purifier. With the exception of e, the components are labelled as for Figure 4. The present purifier contains no superleak. Instead, there is a closely fitting copper sleeve, e, surrounding the flushing tube. The arrows indicate the directions of mass flow. 


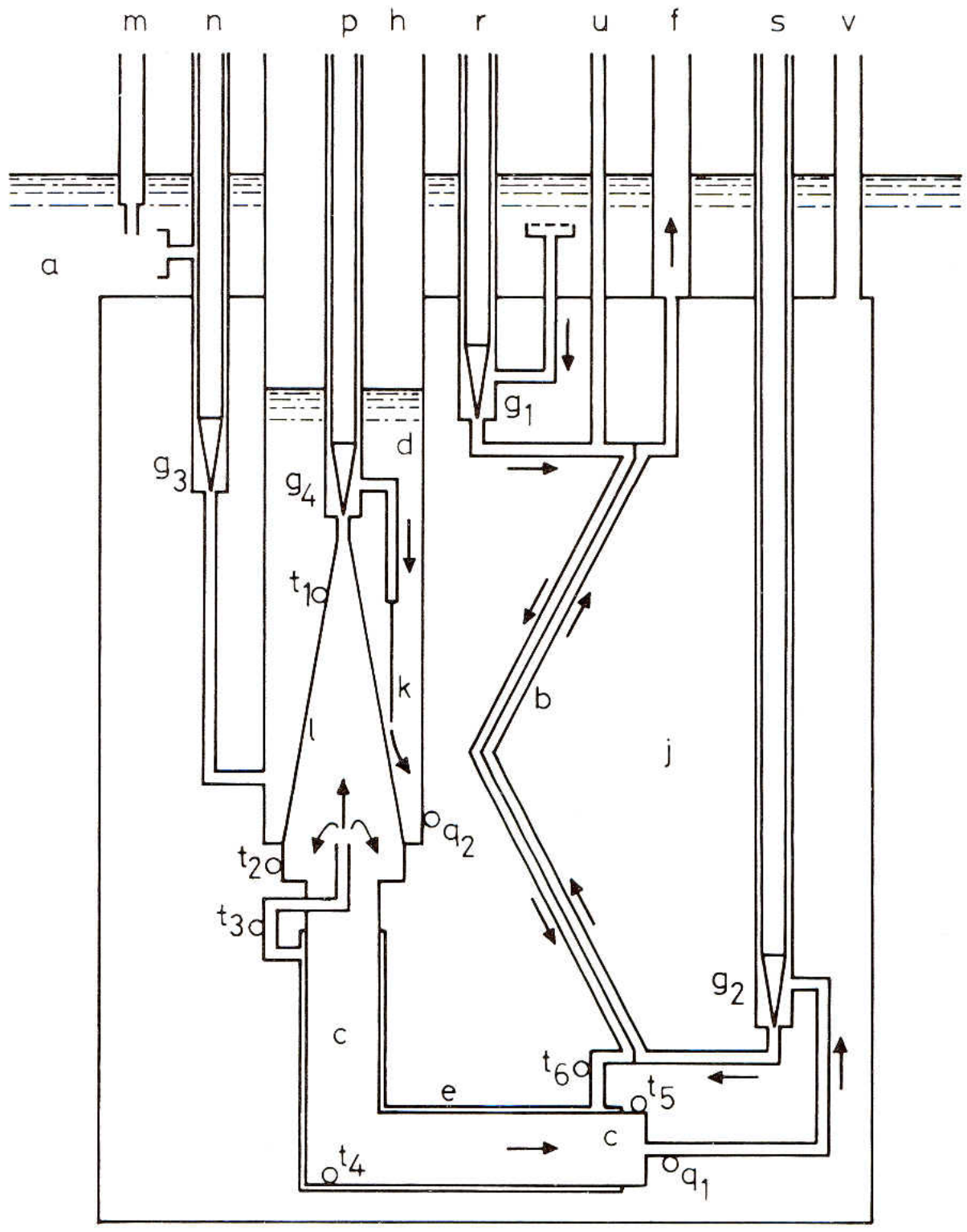

Figure 6: Isotopic purifier (diagrammatic). a, Bath of liquid helium under atmospheric pressure at $4.2 \mathrm{~K}$; b, concentric tube heat exchanger; c, flushing tube; d, pumped bath of He II at = $1.2 \mathrm{~K}$; e, copper sleeve on flushing tube; f, exit tube for isotopically purified 4He; $\mathrm{g}_{1}, \mathrm{~g}_{2} \mathrm{~g}_{3}, \mathrm{~g}_{4}$, needle valves; $\mathrm{h}$, pumping tube for bath, $\mathrm{d} ; \mathrm{j}$, vacuum space; $\mathrm{k}$, restriction; l, copper conical heat exchanger; $\mathrm{m},{ }^{4} \mathrm{He}$ filling siphon; n,p.r,s, needle valve guide tubes; $\mathrm{q}_{1}, \mathrm{q}_{2}$ heaters; $\mathrm{t}_{1}-\mathrm{t}_{6}$, carbon resistance thermometers; $\mathrm{u}$, pump-out tube for purification circuit; v, pump-out tube for vacuum can. 


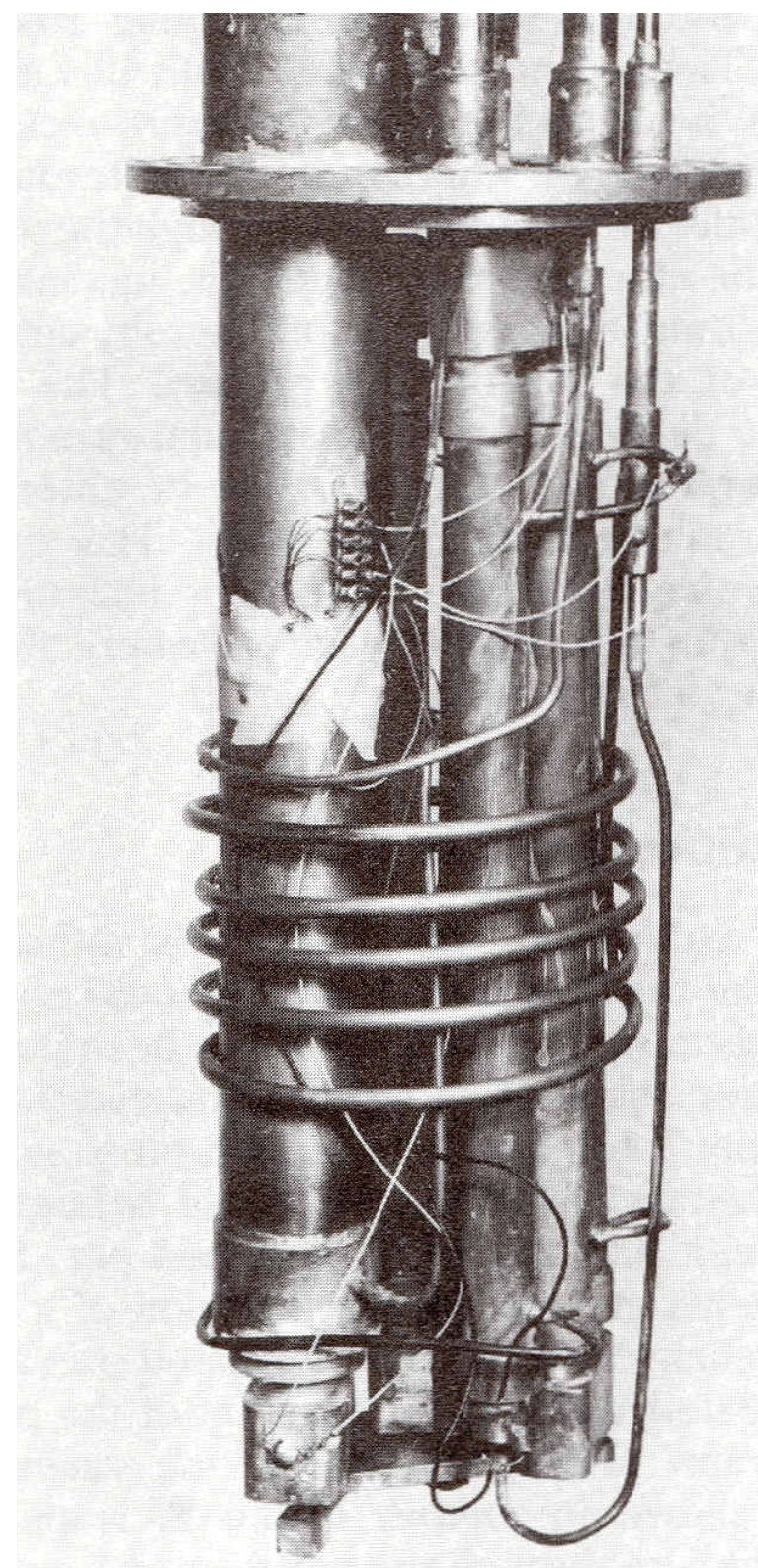

Figure 7: Photograph of the lower portion of the isotopic purification cryostat insert, with the vacuum can removed. The pumped ${ }^{4} \mathrm{He}$ bath (d in Figure 6) is visible on the left of the picture; two sleeved sections of the flushing tube can be seen in the middle and towards the right (e in Figure 6); and one of the needle valves ( $\mathrm{g}_{2}$ in Figure 6) is shown on the extreme right. The concentric tube heat exchanger (b in Figure 6) is coiled around the whole assembly inside the vacuum can, except for the needle valve connecting tubes which, for convenience of assembly and repair, lie outside the heat exchanger. 


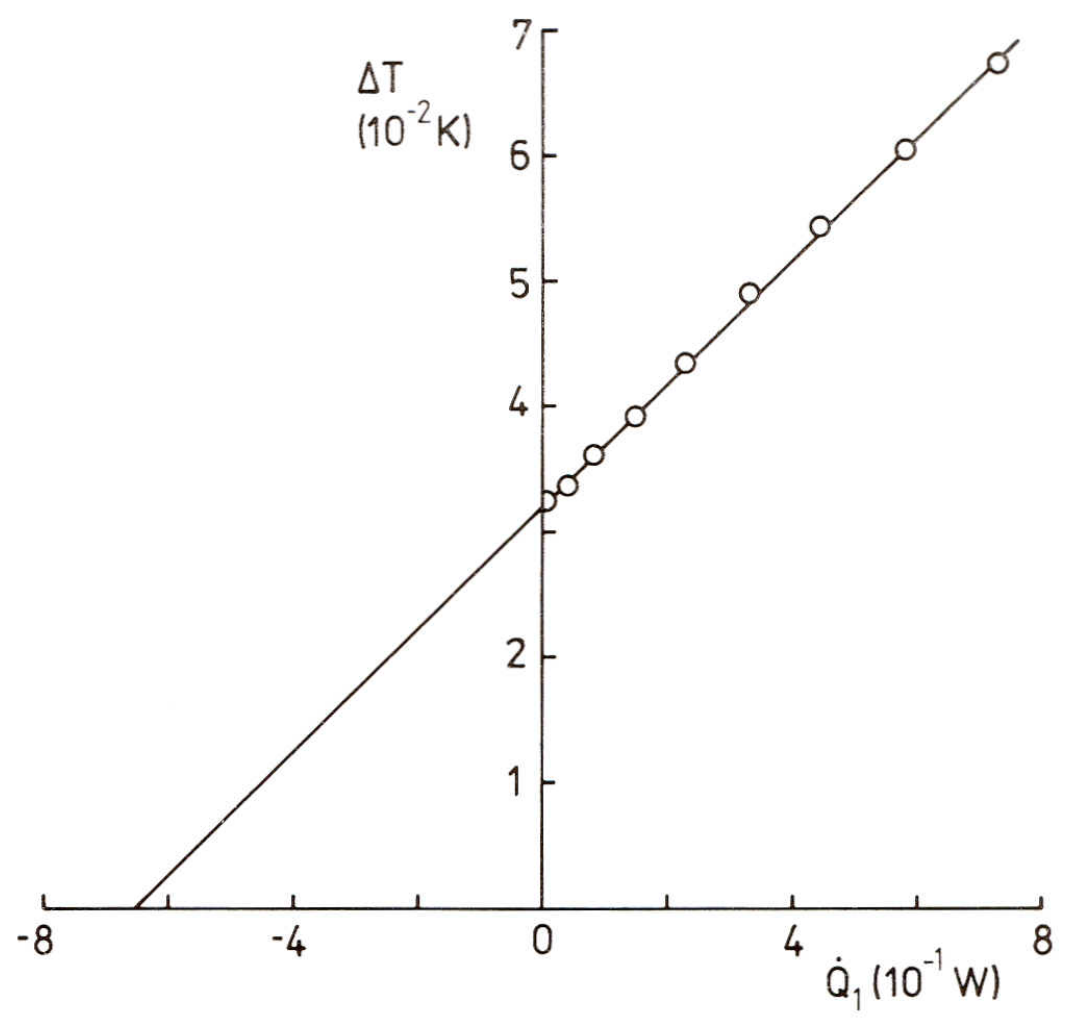

Figure 8: Measured temperature difference, $\Delta T$, across the copper cone (l in Figure 6), plotted as a function of the heater power, $\dot{Q}$ applied to the flushing tube (at heater $\mathrm{q}_{1}$ in Figure 6) for a bath temperature of $1.22 \mathrm{~K}$ 